Original Research

\title{
Internet Mindfulness Meditation Intervention (IMMI) Program with iMINDr Application Support for Older Adults with Depression Symptoms: A Feasibility Study
}

Lutvija Hrnjic ${ }^{*}$, Nina Fry, Helané Wahbeh

Institute of Noetic Sciences, Petaluma, California, USA; E-Mails: Ihrnjic@noetic.org; nfry@noetic.org; hwahbeh@noetic.org

* Correspondence: Lutvija Hrnjic; E-Mail: Ihrnjic@noetic.org

Academic Editor: Peta Stapleton

Special Issue: Mind-Body Approaches that are Revolutionizing the Health Field

OBM Integrative and Complementary Medicine

2021, volume 6 , issue 4

doi:10.21926/obm.icm.2104034
Received: April 16, 2021

Accepted: September 28, 2021

Published: October 14, 2021

\begin{abstract}
The growing population of older adults with depression is a significant public health concern, and effective treatments are necessary. Mindfulness meditation intervention offers effective treatment for depression, but little research has been conducted on the older population. This study aimed to evaluate if the combination of the Internet Mindfulness Meditation Intervention (IMMI) plus iMINDr application improves well-being in older adults with depressive symptoms. Potential participants were recruited online. IMMI included a one-hour online session once a week, a daily 30-minute home practice of guided meditation using the iMINDr app, and a workbook. Measures were collected online before and after the six-week intervention period. Online session adherence was tracked. Thirty-eight participants completed all study requirements and are included in the analysis. Participants showed clinically and statistically significant improvements in depression symptoms, well-being, positive and negative affect, sleep quality, and pain intensity. Participants took $9.9 \pm 3.5$ weeks
\end{abstract}

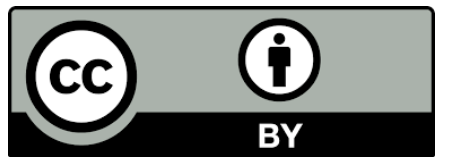

(C) 2021 by the author. This is an open access article distributed under the conditions of the Creative Commons by Attribution License, which permits unrestricted use, distribution, and reproduction in any medium or format, provided the original work is correctly cited. 
to complete the course. High attrition rates mainly were related to participants' motivation to complete the course and stress levels. There were no significant demographic differences between participants and depression symptoms. Potential applications and limitations are discussed. Internet Mindfulness Meditation Intervention (IMMI) was effective in treating depression symptoms in older adults.

\section{Keywords}

Depression in older adults; iMINDr; Internet Mindfulness Meditation Intervention (IMMI); insomnia; pain; positive and negative affect; sleep quality

\section{Introduction}

Older adults are a growing and significant population. The number of US adults 65 years and older grew by $34.2 \%$ in the past ten years [1] and is expected to increase by nearly $50 \%$ from 2016 to 2030. Older adults will likely require support from many society sectors to ensure their physical health and well-being [2], which is why researching alternative treatments such as meditation is essential. The study's objective was to evaluate if a mindfulness meditation internet program in addition to a home practice smartphone application would improve well-being in older adults with depressive symptoms.

Older adults need greater support because they have more outstanding health issues, including mental health conditions [3]. Aging is associated with decreased cognitive function resulting from psychological stress, including depression [4]. Late-life depression can lead to increased mortality rates from medical disorders and suicide if not appropriately treated and is caused by various intricate relationships between social, biological, and psychological components [5]. Older adults' health issues became even more evident with the COVID-19 pandemic stay-at-home and social distancing orders that put older adults at higher risk for suicide [6], and continuing age discrimination in medical care treatment [7].

At present, over 239,000 people have died in the United States from the COVID-19 pandemic, more than $90 \%$ of which are over 50 years old (15.2\% 50-64 years old, $20.9 \%$ 65-74 years old, $26.6 \%$ 75-84 years old, and $32.2 \% 85+$ ) [8]. The COVID-19 highlighted the imperative of finding alternative treatments to help this population now.

Treating depression in older adults is often challenging due to limited prevention initiatives and appropriate diagnosis. Some issues related to early recognition and effective treatment of depression in older adults are: a) older patients rarely report the symptoms due to cognitive decline and a stigma associated with mental illness; b) lack of transportation to medical facilities; c) clinicians' varied adequacy in detection or treatment; d) scarcity of geriatric psychiatrists (one geriatric psychiatrist per 5,682 older patients with a psychiatric condition is estimated by 2030); e) managing fragmented care [9]; and f) increased costs for health care for older adults with depression compared to those without $[4,10,11]$. The recommended treatments for depression in older adults are psychotherapy and medication, but these can be ineffective and cause unwanted side effects such as palpitations, weight gain, and death [12-15]. 
Older adults also suffer from depression-related symptoms like sleep disturbances [14] and chronic pain [15]. Also, people with depressive symptoms have lower levels of self-compassion, which are negatively correlated to depressive symptoms, rumination, and cognitive and behavioral avoidance [16]. People with depression symptoms find that compassion and self-compassion can help them deal with their symptoms [17], and psychotherapies are encouraged to incorporate compassion into their treatments $[16,17]$.

Additional options for delivering effective and safe care to elders with depression symptoms are needed. With the increasing risk of suicide and mortality rates among older adults caused by COVIDand non-COVID-related health conditions such as depression, clinicians and healthcare professionals have a moral imperative for delivering alternative, quick, more effective, safe, and accessible solutions for older adults with depression. The COVID-19 pandemic also highlights the imperative to find alternative treatments that allow them to socially distance at home.

Because of the above issues, mind-body therapies are often sought and used by patients as they are cost-effective, have few or no harmful effects, and empower patients to play an active role in their treatment $[16,17]$. Mindfulness meditation is one such option that helps older adults with their mood symptoms. Previous studies provide evidence that meditation programs improve sleep, stress-related issues, anxiety, pain, and depression in clinical and older adults [17-20] as effectively as evidence-based therapies for older adults with psychiatric diagnoses [21], and are more effective than aerobic or resistance training for clinically depressed older (>65 aged) populations [22].

However, the standard in-person group format of mindfulness-based interventions (MBIs) can be physically demanding for older adults with immobility issues and challenging for those unwilling to engage in a group setting [23]. An online survey investigated the preferred delivery method for MBIs and found that older adults (60-90 years old) favor the internet (43\%) over individual (39\%) or group (10\%) format, and $10 \%$ stated they would not engage in a group format [24]. The online format for MBls could provide one solution to accommodate this population with an alternative to the group format.

The Internet Mindfulness Meditation Intervention (IMMI) is one such program that offers a desirable format and helps with depression, sleep, and pain. IMMI brings mindfulness meditation education and practice to the comfort and safety of people's homes via the Internet through a sixweek program consisting of one-hour weekly sessions and 20 minutes of daily home practice [25]. Previous studies on IMMI's effect on relatively healthy adults reported increased mindfulness home practice engagement than the control group [26]. Using IMMI with older adults has been found satisfactory, feasible, and without adverse effects [27]. The latest study investigating IMMI's effects on older adults (55-80 years old) with depression and related symptoms reported improved sleep disruption, perceived stress, pain intensity, and depression symptoms [23].

The current study builds on previous work to evaluate the effects of IMMI with the addition of a smartphone application for home practice (iMINDr). The study offers a preferred delivery format known to increase meditation practice with comfortable, safe, and convenient access to meditation without requiring participants to visit the research lab and a more accessible home practice platform. The study's objective was to evaluate if the combination of IMMI plus iMINDr effectively improves well-being in older adults with depressive symptoms. Mood and related outcomes collected for this trial were depression symptoms, well-being, affect, compassion, sleep quality, and pain intensity. We hypothesized that the combination of IMMI and iMINDr would decrease depressive symptoms in older adults. 


\section{Methods}

The Institute of Noetic Sciences (IONS) Institutional Review Board (IRB) approved all study activities (WAHH_2018_02).

\subsection{Recruitment}

Older adults (50-90 years old) with depression symptoms were recruited mainly through outreach to IONS' community members, including 1) eNewsletters (membership 65,000); 2) community group-specific eNewsletter ( 85,000 followers); 3) targeted email outreach; 4) blogs, 5) IONS' website; 6) associated social networks; 7) affiliate organizations' social networks; 8) networking sites such as NextDoor and Craigslist, and 9) participants who had previously completed other meditation-related studies with IONS.

\subsection{Screening}

Potential participants were provided with a link to a HIPAA-compliant SurveyMonkey screening questionnaire about inclusion/exclusion criteria, which were kept broad to maximize recruitment, generalizability, and public health applicability. Inclusion criteria were: age 50-90 years old, Center for Epidemiologic Studies Depression Scale-5 (CESD-5) [28] greater than four and less than 32, current residence in the United States, regular access to a smartphone, tablet, or computer, and the internet, and willingness to complete all six IMMI sessions. Exclusions were: significant acute medical illness that would decrease the likelihood of study completion (self-report), significant and untreated depression (CESD-5>32), and previous meditation practice that exceeded 5 minutes a day for 30 or more days in the last six months. Those who were not eligible to participate in the study were provided with mental health care resources and mindfulness meditation resources.

\subsection{Procedures}

Eligible participants were invited to review the Informed Consent, which described the study procedures, risks, benefits, and compensation to complete the study. Participants received $\$ 100$ to complete all study measures (the course, the iMINDr, and pre- and post-surveys). The study team's phone number and email were provided to all participants if they needed assistance with the study or Informed Consent. Once enrolled, participants received the pre-intervention measures and access to the six-week IMMI intervention and iMINDr smartphone application. The self-paced IMMI intervention included three components: 1) a one-hour online video session once a week for six weeks; 2 ) a home practice of daily guided meditation (30 minutes) using the iMINDr app; and 3) an IMMI workbook for tracking participant progress. After participants completed the six-week IMMI course, they received the post-intervention measures.

\section{$2.4 I M M I$}

Once participants completed the pre-intervention measures, they were provided instructions via email to access the six-week IMMI course, the iMINDr app, and the digital IMMI Workbook. Participants were asked to commit to about six-and-a-half hours of practice per week for six weeks. They could practice at any time that was convenient for them, although practicing at the same time 
each day was recommended. The staff involvement was minimal to determine IMMI's effect with the least amount of support.

IMMI's standardized and structured program was designed to increase participant awareness, understand and improve their stress-related reactions, and encourage their inspiration for self-care and a sense of competence and mastery [25]. Each IMMI session contained: 1) educational instruction and discussion on stress, relaxation, meditation, and mind-body relationship; 2) solution-driven methods for successful and joyful mindfulness practice; and 3) formal and informal mindfulness meditation guidance and practice [25]. Formal meditation sessions consisted of a mindful Body Scan and Sitting Meditation (breath awareness, body sensations, cognitive and affective processing). Participants had to complete each online session before continuing to the next one.

Informal practice sessions promoted mindful daily activities (e.g., eating, cleaning, walking) to generalize mindfulness outside of formal practice. Formal practices included daily guided meditations for approximately 30 minutes per day. The iMINDr smartphone application administered the formal guided meditation home practice. The course workbook summarized each session's content and home practice for the week. The research staff did not collect these workbooks.

Upon enrollment in the study, the research managers encouraged participants to set times in their calendars to complete the IMMI Course videos (1-hour per week for six weeks) and complete the daily iMINDr meditations. The research team tracked participant adherence by checking IMMI's administrative portal several times a week to confirm online session completion (attendance). Participants who failed to complete one or more IMMI sessions were contacted up to three times via email or phone to inquire about their continued participation and any additional support they might require to complete the study. Study staff offered email and phone/text reminders to complete the weekly IMMI sessions. If participants did not respond to contact or complete the session after three attempted contacts, they were considered drop-outs. Upon completing the 6week IMMI sessions, participants completed the post-intervention survey. After the survey was complete, they received access to the online guided meditations for a year to continue their practice.

\subsection{Measures}

The following measures were collected through SurveyMonkey.com before and after the six IMMI sessions.

\subsubsection{Well-Being}

The Arizona Integrative Outcomes Scale (AIOS) [29] is a single item, visual-analog self-rating scale that evaluates overall subjective sense of well-being. Participants are asked, "Please reflect on your sense of well-being, taking into account your physical, mental, emotional, social, and spiritual condition over the past 24 hours. Please move the slider below to a point that summarizes your overall sense of well-being for the past 24 hours." The slider is from 0-100 with $0=$ worst you have ever been, and 100 = best you've ever been. The scale results in one value with larger values indicating greater well-being. Convergent and divergent validity was significant compared to the Global Health Index (0.38) and Global Severity Index (-0.41), negative affect (-0.41), and positive affect (0.56) of the Positive and Negative Affect Scale. 


\subsubsection{Depression and Mood-Related Symptoms}

Depression: Center for Epidemiologic Studies Depression Scale is a commonly used self-report measure of depressive symptoms, with a 5 -item subset of the original 20 -item scale (CESD-5). The CESD-5 was used for the screening process. The raw score was multiplied by 4 for cutoff score criteria determination. The CESD- 5 has demonstrated good sensitivity $(>0.84)$, specificity $(\geq 0.80)$, and high validity $(>0.90)$ for identifying current depression symptoms by the full 20-item scale [30]. The full CESD was used for evaluating depression symptoms before and after the intervention. Participants reported values for each item (e.g., depressed mood, movement/exercise, loss of appetite, sleeping problems, feelings of guilt, worthlessness, and helplessness), producing total scores ranging from 0 - 60, where higher scores reflect greater depression symptoms [31].

Affect: The Positive and Negative Affect Schedule (PANAS) is a self-report measure including two mood scales, one measuring positive affect and the other measuring negative affect [30, 31]. Users rate each item on a 5-point Likert Scale ranging from $1=$ never to $5=$ always. Five items measure positive affect, and five items measure negative affect. Positive and negative items are summed for the scores. The scales range from $5-25$, with higher scores representing higher positive or negative affect levels.

Compassion: Dispositional Positive Emotions Scale Compassion subscale is a five-item scale asking participants about their capacity for compassion and compassionate acts rated on a Likert Scale from 1 = strongly disagree to 7 = strongly agree [32]. Compassion is an emotional response when suffering is perceived and involves an authentic desire to alleviate that suffering. Higher scores indicate greater levels of positive compassion.

\subsubsection{Depression-Related Symptoms}

Sleep Quality: Acute sleep is a single-item 11-point numeric scale measuring acute sleep. Participants rate their sleep quality over the past 24 hours on a scale ranging from $0-10$, with $0=$ best possible sleep and $10=$ worst possible sleep. An investigation of this Sleep Quality Scale suggests the single-item format has favorable measurement properties. Specifically, the results provide evidence of its reproducibility, convergent validity, and responsiveness to treatment as an overall sleep rating in two clinical trials [33].

Pain: The Numeric Pain Rating Scale (NPRS) is a visual analog scale in which a respondent rates the intensity of their pain "in the last 24 hours," or average pain intensity. The 11-point numeric scale ranges from $0=$ no pain to $10=$ worst possible pain. The NPRS is a reliable scale in terms of inter- or intra-rater repeatability and its ability to detect change [34].

\subsubsection{Subjective Experience of Intervention}

Transformation items: After the intervention, participants rated how true they felt the following statement was, "I feel like I have positively changed as a result of this workshop," on a scale of 0 100 with $0=$ definitely false and $100=$ definitely true. They also rated if the following statement was true, "I experienced a moment of clarity or profound insight during my workshop."

Open-ended text: There was also the optional question, "Is there anything you would like us to know about your workshop/event experience?" where participants could type their open-ended answers. These comments were qualitatively reviewed. 


\subsection{Statistical Analysis}

Means, standard deviations are reported for continuous variables and counts and percentages for categorical variables. Data manipulation and analyses were performed using the statistical programming language $R$ [35] and STATA 15.1. The raw data were analyzed by taking the difference of pre- and post-event scores (pre- minus post-). The normality of the differences was assessed using the Shapiro-Wilk test. Non-normally distributed differences were analyzed using the Wilcoxon signed-rank test if symmetric and the Sign test otherwise.

Data were imputed using the R package Amelia [36], which performs multiple imputation on cross-section (survey) or time-series data. If both pre- and post-event data were missing for a participant, the observation was removed (as it cannot be accommodated by multiple imputation). 100 imputed data sets were generated per t-test. Paired t-tests were performed using the R package MKmisc [37] using functions specifically designed to perform multiple imputation Student's t-tests (t-tests that are carried out on multiple imputed data sets). This procedure was repeated once for only completers and once for both completers and non-completers. A False Discovery Rate correction for multiple comparisons was applied using $p=0.05$ as the cutoff [38].

The Cochrane Handbook for Systematic Reviews of Interventions [39] states that intention-totreat analysis can be performed in the presence of missing data using imputation, wherein sensitivity analysis involves the use of different methods to validate the result. This approach is used here to generate three sets of results for validation.

\section{Results}

\subsection{Participants}

Study activities occurred between April 10, 2019, and February 11, 2020. With extensive recruitment, 389 people accessed the screening questionnaire. Of those, eight stopped after the consent form, 13 were younger than 50, 12 did not have a smartphone, 71 had more than 5 minutes of daily meditation practice, two stopped after the meditation question, and 47 did not leave any contact information. That resulted in 228 volunteers who were invited to complete the CESD depression screening questionnaire, 220 of whom completed it. Of those, 154 were eligible and asked to complete the baseline measures (66 were not eligible because their CESD score was less than five or greater than 31). One hundred and four participants completed the baseline survey, 102 of which responded and received the intervention materials (e.g., log-in information and instructions). Of the 102, 19 dropped out for various reasons (i.e., one cancer diagnosis, one ill spouse, four too busy, four technical issues or didn't like the program, nine no explanation). Seven of the people who dropped out completed module 1, and two completed module 2. Two participants completed all six modules in 45 minutes. Forty did not respond to the three staff reminders. Thirty-eight participants completed all six IMMI sessions. All data was retained for the intention-to-treat analysis. Participant characteristics are displayed in Table 1. Although participants were requested to complete one session per week, the average time to complete the course was $9.9 \pm 3.5$ weeks (range 4-22). 
Table 1 Participant characteristics.

\begin{tabular}{lll}
\hline Characteristics & Units/Categories & $\begin{array}{l}\text { Values } \\
\text { Mean (SD) or n (\%) }\end{array}$ \\
\hline Age & Years & $63.3 \pm 8.0$, range 50-83 \\
Education & Years & $16.9 \pm 3.3$ \\
Gender & Female & $74(71.2)$ \\
& Male & $30(28.9)$ \\
Ethnicity & Asian or Asian American & $3(2.9)$ \\
& Black or African American & $3(2.9)$ \\
& Hispanic & $2(2.0)$ \\
Relationship & White/Caucasian & $95(91.4)$ \\
& Other & $1(1.0)$ \\
& In a relationship & $68(66.7)$ \\
Setting & Not in a relationship & $34(33.3)$ \\
& No response & $2(5.3)$ \\
& Rural & $19(18.6)$ \\
& Suburban & $54(52.9)$ \\
& Urban & $29(28.4)$ \\
& Poor & $1(1.0)$ \\
& Fair & $13(12.6)$ \\
& Good & $30(48.5)$ \\
& Very good & $3(2.9)$ \\
\hline
\end{tabular}

Note. $n=104$.

\subsection{Mood and Related Outcomes}

After comparing the before and after IMMI participants' data, there were improvements in depression symptoms, well-being, positive and negative affect, sleep quality, and pain intensity (Table 2). Most participants felt that the statement, "I feel like I have positively changed due to this workshop," was true $(81.6 \pm 19.6)$. Seventy-six percent of the participants endorsed the statement, "I experienced a moment of clarity or profound insight during my workshop."

Table 2 Depression and related symptoms summary statistics and paired test results for the data before and after IMMI + iMINDr without multiple imputation.

\begin{tabular}{llllllll}
\hline \multirow{2}{*}{ Outcome } & Pre & Pre & Post & Post & $\Delta$ & $\Delta$ \\
& $\mathrm{n}$ & Mean (SD) & $\mathrm{n}$ & Mean (SD) & $\mathrm{n}$ & Mean (SD) & Paired Test Result \\
\hline Depression $^{\mathrm{a} *}$ & 104 & $20.7(8.5)$ & 41 & $13.3(8.8)$ & 41 & $7.4(10.2)$ & $\mathrm{t}(40)=4.7, \mathrm{p}=0.00003$ \\
\hline
\end{tabular}




\begin{tabular}{|c|c|c|c|c|c|c|c|}
\hline Well-Being ${ }^{a *}$ & 97 & $51.8(16.8)$ & 41 & $66.0(17.8)$ & 38 & $\begin{array}{l}-14.1 \\
(17.7)\end{array}$ & $\begin{array}{l}t(37)=-4.971, \quad p= \\
0.00002\end{array}$ \\
\hline Positive Affect ${ }^{a *}$ & 100 & $15.7(3.6)$ & 41 & $17.4(4.3)$ & 41 & $-2.1(4.7)$ & $t(40)=-2.87, p=0.007$ \\
\hline Negative Affect ${ }^{\mathrm{b} *}$ & 100 & $10.2(3.4)$ & 41 & $8.4(3.5)$ & 41 & $1.1(4.2)$ & $V=453, p=0.02$ \\
\hline Compassion $^{c}$ & 103 & $5.3(1.2)$ & 42 & $5.7(1.1)$ & 42 & $-0.2(0.8)$ & $s=15, p=0.50$ \\
\hline Sleep Quality ${ }^{a *}$ & 102 & $4.7(2.3)$ & 42 & $2.9(2.2)$ & 42 & $2.1(2.6)$ & $\mathrm{t}(41)=5.3, \mathrm{p}=0.000005$ \\
\hline Pain ${ }^{c *}$ & 98 & $3.6(2.7)$ & 42 & $2.5(2.4)$ & 38 & $1.0(2.1)$ & $s=18, p=0.03$ \\
\hline
\end{tabular}

Note. Affect $(5-25 ; 25=$ higher levels of positive or negative affect); Compassion (1-7; 7 = greater levels of compassion); Depression (0-60; 0 = no depression symptoms); Pain last 24 hours (0-10; $0=$ no pain); SD-Standard Deviation; Sleep quality last night $(0-10 ; 0=$ best sleep); WellBeing/AIOS (0-1000; 0 = worst you have ever been).

${ }^{\mathrm{a}}=$ paired t-test, ${ }^{\mathrm{b}}=$ Wilcoxon Signed Rank Test, ${ }^{\mathrm{c}}=$ Sign-Test; *Significant using False Discovery Rate $p=0.05$

Multiple imputation of the completer analysis (Table 3 ) revealed similar results for depression and well-being. The pain measure $p$-value became more significantly significant $(p=0.03$ without imputation and $p=0.009$ with imputation).

Table 3 Summary statistics and paired t-test results for completers with multiple imputation.

\begin{tabular}{lll}
\hline Outcome & $\begin{array}{l}\Delta \\
\text { Mean (SD) }\end{array}$ & Paired Test Result \\
\hline Depression* & $7.5(10.2)$ & $\mathrm{t}(38)=4.7, \mathrm{p}=0.00003$ \\
Well-Being* & $-13.9(18.2)$ & $\mathrm{t}(37)=-4.9, \mathrm{p}=0.00002$ \\
Pain* & $0.9(2.2)$ & $\mathrm{t}(36)=2.8, \mathrm{p}=0.00002$ \\
\hline
\end{tabular}

Note: $\mathrm{n}=42$ Measures that are not displayed here did not have any missing data or had instances of both pre- and post-survey values missing for a participant that were removed (these are not accommodated by the imputation procedure). *Significant using False Discovery Rate $p=0.05$

Multiple imputation of completer and non-completer data (Table 4) also revealed similar results with all measures except for compassion being significantly improved even with multiple comparison corrections.

Table 4 Summary statistics and paired t-test results for completers and non-completers with imputation.

\begin{tabular}{llll}
\hline Outcome & $\Delta$ & $\Delta$ & Multiple Imputation
\end{tabular}




\begin{tabular}{lcll}
\hline & $\mathrm{n}$ & Mean (SD) & Paired Test Result \\
\hline Depression* & 104 & $7.4(14.5)$ & $\mathrm{t}(51)=5.2, \mathrm{p}=0.000003$ \\
Well-Being* & 100 & $-14.1(26.2)$ & $\mathrm{t}(39)=-5.4, \mathrm{p}=0.000003$ \\
Positive Affect* & 100 & $-1.8(6.7)$ & $\mathrm{t}(41)=-2.7, \mathrm{p}=0.01$ \\
Negative Affect* & 100 & $1.5(6.0)$ & $\mathrm{t}(45)=2.5, \mathrm{p}=0.02$ \\
Compassion & 103 & $-0.2(1.4)$ & $\mathrm{t}(32)=-1.7, \mathrm{p}=0.09$ \\
Sleep Quality* & 102 & $1.9(3.3)$ & $\mathrm{t}(49)=5.9, \mathrm{p}<0.0000005$ \\
Pain* & 102 & $1.0(3.1)$ & $\mathrm{t}(40)=3.3, \mathrm{p}=0.002$ \\
\hline
\end{tabular}

Note: $\mathrm{n}=42$ Measures that are not displayed here did not have any missing data or had instances of both pre- and post-survey values missing for a participant that were removed (these are not accommodated by the imputation procedure). *Significant using False Discovery Rate $p=0.05$

\subsection{Subjective Experience of Intervention}

Most participants felt that the statement, "I feel like I have positively changed due to this workshop," was true $(81.6 \pm 19.6)$. Seventy-six percent of the participants endorsed the statement, "I experienced a moment of clarity or profound insight during my workshop." After the intervention survey included an open-text field requesting feedback about the study, twenty-one participants responded, and fifteen participants wrote a positive comment about the course or how it affected them. For example, some participants commented on the program's positive impact on their wellbeing "... despite a challenging life situation presently...," and "... helpful with everyday challenges, and for finding great value in it."

Others-reported that the coping skills they learned in this program were helpful in stressful situations, and that friends and family also noticed their "new-found equilibrium."

One participant specifically attributed the improvement in health to this practice after the doctor confirmed that their "... regular blood test numbers were really good... especially... blood pressure."

Two participants suggested improvements in audio quality and another in the course structure. Three participants talked about personal difficulties in their lives that precluded them from successfully participating in the course.

\section{Discussion}

\subsection{Summary}

The current study examined the combined effects of an Internet Mindfulness Meditation Intervention (IMMI) program and iMINDr app on mood and related outcomes for older adults with depression symptoms with minimal research staff support. The completion rate of people initially enrolled in the study was low (24\%). The study was feasible in that interested participants were able to complete the study activities given enough time. Depression symptoms, general well-being, 
higher positive and lower negative affect, sleep quality, and pain levels improved after completing the IMMI intervention.

\subsection{Participant Characteristics}

There were no critical demographic differences between participants and depression symptoms. Most participants were Caucasian, which aligns with nationally representative survey findings [40]. More women took part in this study, as is the case with most complementary and alternative medicine studies [41]. All the participants were novice meditators.

\subsection{Adherence}

Participants had to complete all six sessions to be considered completers, and so all included participants were exposed to the six sessions. In general, participants did not have any issues downloading the iMINDr application for android or iOS devices. Increasingly older adults are now comfortable with smartphone technology, as reflected by a recent Pew Research Center survey reporting that $42 \%$ of US adults 65 and older and 59\% of 65-69-year olds use smartphones [42]. Having participants download the iMINDr app on their smartphones rather than mailing them a study iPod, as was done in previous studies, was a vast logistical improvement helpful for future studies. Those studies focused on the objective data collected by iMINDr to observe practice patterns in general and in relation to outcomes.

High attrition rates were mostly related to participants' lack of motivation to complete the course and stress levels. We encountered similar issues in this study to other meditation studies in older adults [21, 27]. We made minimal efforts to support adherence to the study activities, as was the study's purpose. The contact we did make was helpful with the participants who were committed to completing the program. Additional improvements could be to make the course more engaging or motivating so that participants would be inspired to complete it despite limited encouragement and high-stress levels.

We found that eligible participants were initially very eager to participate in the study intervention but had trouble completing the study components. Likely, the combination of depression-related symptoms and some challenges with technology in this older population presented obstacles to complete self-directed study activities, such as watching the 1-hour weekly IMMI course material or meditating daily. Additionally, participants were often struggling with life circumstances, such as health issues, caring for ailing parents or loved ones, or coping with California wildfire-related issues that impeded their ability to dedicate time to the study. Motivated participants completed all six online sessions with minimal support if they had lower stress levels at home or their motivation levels were high enough to overcome the barriers for them to participate fully in the program. Future studies that aim to maximize participant adherence should consider adding more staff support (e.g., scheduled email/text reminders and telephone interactions).

\subsection{Mood and Related Outcomes}

According to the Center for Epidemiological Studies Depression Scale (CESD) scoring guide, the IMMI participants showed a statistically significant and clinically important improvement from before to after the IMMI course as found in other studies as well [23]. General well-being also 
improved, as other mindfulness studies have also found [43]. Sleep quality also improved clinically and statistically in the IMMI participants. These improvements reflect previous findings in older adults with depression [23] and patients with insomnia and other chronic conditions [39-41]. Pain intensity scores improved significantly upon the IMMI completion but not as much as depression, well-being, affect, and sleep. Previous studies also report a positive influence of mindfulness meditation on pain outcomes [42-47], although the improvement may be due to the effect of the psychological aspect of chronic pain [48]. Improved depression, well-being, affect, sleep quality, and pain in participants with depression and related outcomes are noteworthy, considering that participants had minimal research staff support and were novice meditators.

\subsection{Limitations}

Several limitations should be considered when interpreting the results. The primary limitation is the lack of a control group. Future studies should consider a well-matched control group to evaluate effects. Our results are based on self-reported data, and future studies should be supplemented with objective methods [49]. Favorable correlations between the measures of well-being and social desirability have been found in previous studies [50] and should be taken into consideration when interpreting the results of our study since our participants received monetary compensation for their time. Furthermore, no cognitive screen or measure was included. High attrition may also have been from any cognitive decline participants were experiencing that prevented them from successfully navigating the technology requirements of the study. We did not measure expectancy and credibility, and future studies should consider including them as participants' expectations toward the meditation practice may play a significant role in self-reported improvements. Although the benefits of mind-body interventions do not depend on expectancy effects [51], measuring this outcome is still recommended for better understanding and maximum impact of similar trials [52]. Even though having mostly Caucasian women prevented the results' generalizability, these demographics reflect previous complementary and alternative medicine studies [41]. High attrition rates were expected, as is usually the case in studies with minimal staff support $[53,54]$ and yet, introduces a high risk of bias. The intention to treat analyses attempt to mitigate this risk and they did not find any substantive differences in the results.

\subsection{Future Directions}

Future studies would benefit from having an active and passive control group, additional timepoints of data collection to examine sustained positive effects of mindfulness meditation on depression, and additional staff support to increase adherence.

\section{Conclusions}

In conclusion, older adults who completed the IMMI course showed improvements in depression, well-being, affect, sleep quality, and pain symptoms. IMMI provides easy access and safe delivery of low-dose and -cost mindfulness meditation intervention via the internet, available to the older population who are unable to commute, do not like group settings, and need safe and effective therapies when interpersonal or professional help is unavailable, during and outside of the COVID19 pandemic. 


\section{Acknowledgments}

The authors would like to thank the Mental Insight Foundation, Institute of Noetic Sciences and its supporters, IONS Earthrise Campus Retreat Center staff, Steven Swanson, Alicia Soliz, Maria Wojakowski, and the participants for their contributions to this project.

\section{Author Contributions}

Lutvija Hrnjic: Writing-Original Draft Preparation; Writing-Review and Editing. Nina Fry: Methodology; Project Administration; Recruitment and Study Adherence; Supervision; Data Curation; Writing-Review and Editing. Helané Wahbeh: Funding Acquisition; Conceptualization; Methodology; Data Curation; Formal Analysis; Supervision; Writing-Review and Editing.

\section{Funding}

This study was funded by the Mental Insight Foundation, California, USA. The funders had no role in the study's design; in the collection, analyses, or interpretation of data; in the writing of the manuscript, or in the decision to publish the results.

\section{Competing Interests}

The authors have declared that no competing interests exist.

\section{References}

1. Bureau UC. 65 and older population grows rapidly as baby boomers age [Internet]. Suitland: The United States Census Bureau; 2020 [cited 2020 September 5th]. Available from: https://www.census.gov/newsroom/press-releases/2020/65-older-population-grows.html.

2. Medina L, Sabo S, Vespa J. Living longer: Historical and projected life expectancy in the United States, 1960 to 2060 [Internet]. Suitland: United States Census Burea; 2020 [cited 2020 September 20th]. Available from: https://www.census.gov/content/dam/Census/library/publications/2020/demo/p251145.pdf.

3. Luo MS, Chui EW, Li LW. The longitudinal associations between physical health and mental health among older adults. Aging Ment Health. 2020; 24: 1990-1998.

4. Oken BS, Chamine I, Wakeland W. A systems approach to stress, stressors and resilience in humans. Behav Brain Res. 2015; 282: 144-154.

5. Aziz R, Steffens DC. What are the causes of late-life depression? Psychiatr Clin. 2013; 36: 497516.

6. Santini ZI, Jose PE, Cornwell EY, Koyanagi A, Nielsen L, Hinrichsen C, et al. Social disconnectedness, perceived isolation, and symptoms of depression and anxiety among older Americans (NSHAP): A longitudinal mediation analysis. Lancet Public Health. 2020; 5: e62-e70.

7. Emanuel EJ, Persad G, Upshur R, Thome B, Parker M, Glickman A, et al. Fair allocation of scarce medical resources in the time of COVID-19. N EngI J Med. 2020; 382: 2049-2055.

8. Centers for Disease Control and Prevention. COVID-19 cases, deaths, and trends in the US | CDC COVID data tracker [Internet]. Atlanta: Centers for Disease Control and Prevention; 2020 [cited 
2020 November 25th]. Available from: https://covid.cdc.gov/covid-data-tracker.

9. Ellison JM, Kyomen HH, Harper DG. Depression in later life: An overview with treatment recommendations. Psychiatr Clin. 2012; 35: 203-229.

10. Cuijpers P, Karyotaki E, Eckshtain D, Ng MY, Corteselli KA, Noma H, et al. Psychotherapy for depression across different age groups: A systematic review and meta-analysis. JAMA Psychiatry. 2020; 77: 694-702.

11. Milan R, Vasiliadis HM. The association between side effects and adherence to antidepressants among primary care community-dwelling older adults. Aging Ment Health. 2020; 24: 12291236.

12. Sobieraj DM, Martinez BK, Hernandez AV, Coleman Cl, Ross JS, Berg KM, et al. Adverse effects of pharmacologic treatments of major depression in older adults. J Am Geriatr Soc. 2019; 67: 1571-1581.

13. Alonso-Pedrero L, Bes-Rastrollo M, Marti A. Effects of antidepressant and antipsychotic use on weight gain: A systematic review. Obes Rev. 2019; 20: 1680-1690.

14. Perach R, Allen CK, Kapantai I, Madrid-Valero JJ, Miles E, Charlton RA, et al. The psychological wellbeing outcomes of nonpharmacological interventions for older persons with insomnia symptoms: A systematic review and meta-analysis. Sleep Med Rev. 2019; 43: 1-13.

15. Fishbain DA, Cutler R, Rosomoff HL, Rosomoff RS. Chronic pain-associated depression: Antecedent or consequence of chronic pain? A review. Clin J Pain. 1997; 13: 116-137.

16. Krieger T, Altenstein D, Baettig I, Doerig N, Holtforth MG. Self-compassion in depression: Associations with depressive symptoms, rumination, and avoidance in depressed outpatients. Behav Ther. 2013; 44: 501-513.

17. Pauley G, McPherson S. The experience and meaning of compassion and self-compassion for individuals with depression or anxiety. Psychol Psychother. 2010; 83: 129-143.

18. Wahbeh H, Elsas SM, Oken BS. Mind-body interventions: Applications in neurology. Neurology. 2008; 70: 2321-2328.

19. Goyal M, Singh S, Sibinga EM, Gould NF, Rowland-Seymour A, Sharma R, et al. Meditation programs for psychological stress and well-being: A systematic review and meta-analysis. JAMA Intern Med. 2014; 174: 357-368.

20. Black DS, O'Reilly GA, Olmstead R, Breen EC, Irwin MR. Mindfulness meditation and improvement in sleep quality and daytime impairment among older adults with sleep disturbances: A randomized clinical trial. JAMA Intern Med. 2015; 175: 494-501.

21. Goldberg SB, Tucker RP, Greene PA, Davidson RJ, Wampold BE, Kearney DJ, et al. Mindfulnessbased interventions for psychiatric disorders: A systematic review and meta-analysis. Clin Psychol Rev. 2018; 59: 52-60.

22. Miller KJ, Gonçalves-Bradley DC, Areerob P, Hennessy D, Mesagno C, Grace F. Comparative effectiveness of three exercise types to treat clinical depression in older adults: A systematic review and network meta-analysis of randomised controlled trials. Ageing Res Rev. 2020; 58: 100999.

23. Wahbeh $\mathrm{H}$. Internet mindfulness meditation intervention (IMMI) improves depression symptoms in older adults. Medicines. 2018; 5: 119.

24. Wahbeh H, Svalina MN, Oken BS. Group, one-on-one, or internet? Preferences for mindfulness meditation delivery format and their predictors. Open Med J. 2014; 1: 66-74.

25. Wahbeh H, Lane JB, Goodrich E, Miller M, Oken BS. One-on-one mindfulness meditation 
trainings in a research setting. Mindfulness. 2014; 5: 88-99.

26. Wahbeh $\mathrm{H}$, Oken BS. Internet mindfulness meditation intervention for the general public: Pilot randomized controlled trial. JMIR Ment Health. 2016; 3: e37.

27. Wahbeh $\mathrm{H}$, Goodrich E, Oken BS. Internet-based mindfulness meditation for cognition and mood in older adults: A pilot study. Altern Ther Health Med. 2016; 22: 44-53.

28. Eaton WW, Smith C, Ybarra M, Muntaner C, Tien A. Center for epidemiologic studies depression scale: review and revision (CESD and CESD-R). In: The Use of Psychological Testing for Treatment Planning and Outcomes Assessment. Mahwah, NJ: Lawrence Erlbaum; 2004. pp.363377.

29. Bell IR, Cunningham V, Caspi O, Meek P, Ferro L. Development and validation of a new global well-being outcomes rating scale for integrative medicine research. BMC Complement Altern Med. 2004; 4: 1.

30. Bohannon RW, Maljanian R, Goethe J. Screening for depression in clinical practice: Reliability and validity of a five-item subset of the CES-Depression. Percept Mot Skills. 2003; 97: 855-861.

31. Radloff LS. The CES-D scale: A self-report depression scale for research in the general population. Appl Psychol Meas. 1977; 1: 385-401.

32. Shiota MN, Keltner D, John OP. Positive emotion dispositions differentially associated with big five personality and attachment style. J Posit Psychol. 2006; 1: 61-71.

33. Cappelleri JC, Bushmakin AG, McDermott AM, Sadosky AB, Petrie CD, Martin S. Psychometric properties of a single-item scale to assess sleep quality among individuals with fibromyalgia. Health Qual Life Outcomes. 2009; 7: 54.

34. Bijur PE, Silver W, Gallagher EJ. Reliability of the visual analog scale for measurement of acute pain. Acad Emerg Med. 2001; 8: 1153-1157.

35. R Development Core Team R. R: A language and environment for statistical computing [Internet]. Vienna: R Foundation for Statistical Computing; 2021. Available from: http://www.Rproject.org/.

36. Honaker J, King G, Blackwell M. Amelia II: A program for missing data. J Stat Softw. 2011; 45: 147.

37. Kohl M. MKmisc: Miscellaneous functions from M. Kohl. R package version 0.993 [Internet]. Furtwangen: WordPress; 2013. Available from: http://www.stamats.de.

38. Benjamini Y, Hochberg Y. Controlling the false discovery rate: A practical and powerful approach to multiple testing. J R Stat Soc Series B Stat Methodol. 1995; 57: 289-300.

39. 16.2.1 Introduction. Cochrane Handbook for Systematic Reviews of Interventions [Internet]. London: Cochrane; 2021 [cited 2021 September 13th]. Available from: https://handbook-51.cochrane.org/chapter 16/16 21 introduction.htm.

40. Cramer H, Hall H, Leach M, Frawley J, Zhang $Y$, Leung B, et al. Prevalence, patterns, and predictors of meditation use among US adults: A nationally representative survey. Sci Rep. 2016; 6: 36760.

41. Barnes PM, Bloom B, Nahin RL. Complementary and alternative medicine use among adults and children; United States, 2007. Atlanta, GA: Centers for Disease Control and Prevention; 2008; Natl Health Stat Rep no. 12.

42. Anderson M, Perrin A. Technology use among seniors [Internet]. Washington: Pew Research Center: Internet, Science \& Tech; 2017 [cited 2020 October 15th]. Available from: https://www.pewresearch.org/internet/2017/05/17/technology-use-among-seniors/. 
43. Bostock S, Crosswell AD, Prather AA, Steptoe A. Mindfulness on-the-go: Effects of a mindfulness meditation app on work stress and well-being. J Occup Health Psychol. 2019; 24: 127-138.

44. Rusch HL, Rosario M, Levison LM, Olivera A, Livingston WS, Wu T, et al. The effect of mindfulness meditation on sleep quality: A systematic review and meta-analysis of randomized controlled trials. Ann N Y Acad Sci. 2019; 1445: 5-16.

45. Neuendorf R, Wahbeh H, Chamine I, Yu J, Hutchison K, Oken BS. The effects of mind-body interventions on sleep quality: A systematic review. Evid Based Complementary Altern Med. 2015; 2015: 902708.

46. Barrett B, Harden CM, Brown RL, Coe CL, Irwin MR. Mindfulness meditation and exercise both improve sleep quality: Secondary analysis of a randomized controlled trial of community dwelling adults. Sleep Health. 2020; 6: 804-813.

47. Zorn J, Abdoun O, Bouet R, Lutz A. Mindfulness meditation is related to sensory-affective uncoupling of pain in trained novice and expert practitioners. Eur J Pain. 2020; 24: 1301-1313.

48. Ball EF, Nur Shafina Muhammad Sharizan E, Franklin G, Rogozińska E. Does mindfulness meditation improve chronic pain? A systematic review. Curr Opin Obstet Gynecol. 2017; 29: 359-366.

49. Baer R. Assessment of mindfulness by self-report. Curr Opin Psychol. 2019; 28: 42-48.

50. Caputo A. Social desirability bias in self-reported well-being measures: Evidence from an online survey*. Univ Psychol. 2017; 16: 245-255.

51. Hicks $M$, Hanes D, Wahbeh H. Expectancy effect in three mind-body clinical trials. J Evid Based Complementary Altern Med. 2016; 21: NP103-NP109.

52. Oken BS. Placebo effect: Clinical perspectives and potential mechanisms. New York: The Parthenon Publishing Group; 2004.

53. Gu Q, Hou JC, Fang XM. Mindfulness meditation for primary headache pain: A meta-analysis. Chin Med J. 2018; 131: 829-838.

54. Hilton L, Hempel S, Ewing BA, Apaydin E, Xenakis L, Newberry S, et al. Mindfulness meditation for chronic pain: Systematic review and meta-analysis. Ann Behav Med. 2017; 51: 199-213.

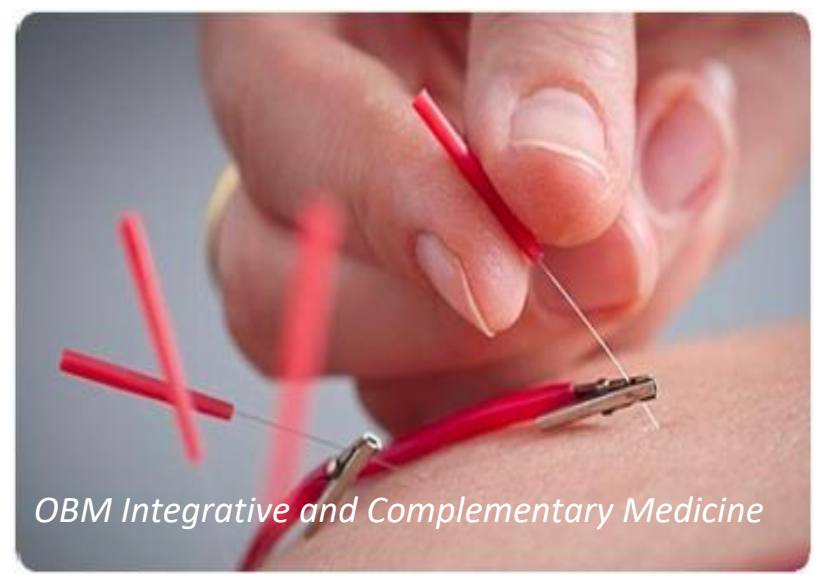

Enjoy OBM Integrative and Complementary Medicine by:

1. Submitting a manuscript

2. Joining in volunteer reviewer bank

3. Joining Editorial Board

4. Guest editing a special issue

For more details, please visit: http://www.lidsen.com/journals/icm 